\title{
Colonization of Enteroaggregative Escherichia coli and Shiga toxin-producing Escherichia coli in chickens and humans in southern Vietnam
}

Nguyen Vinh Trung ${ }^{1,2,3^{*}}$, Hoang Ngoc Nhung ${ }^{3}$, Juan J. Carrique-Mas ${ }^{3,4}$, Ho Huynh Mai ${ }^{5}$, Ha Thanh Tuyen ${ }^{3}$, James Campbell ${ }^{3,4}$, Nguyen Thi Nhung ${ }^{3}$, Pham Van Minh ${ }^{3}$, Jaap A. Wagenaar ${ }^{6,7}$, Nguyen Thi Nhu Mai ${ }^{8}$, Thai Quoc Hieu, ${ }^{5}$ Constance Schultsz ${ }^{1,2,3}$ and Ngo Thi Hoa,

\begin{abstract}
Background: Enteroaggregative (EAEC) and Shiga-toxin producing Escherichia coli (STEC) are a major cause of diarrhea worldwide. E. coli carrying both virulence factors characteristic for EAEC and STEC and producing extended-spectrum beta-lactamase caused severe and protracted disease during an outbreak of E. coli O104:H4 in Europe in 2011. We assessed the opportunities for E. coli carrying the aggR and stx genes to emerge in 'backyard' farms in south-east Asia.

Results: Faecal samples collected from 204 chicken farms; 204 farmers and 306 age- and gender-matched individuals not exposed to poultry farming were plated on MacConkey agar plates with and without antimicrobials being supplemented. Sweep samples obtained from MacConkey agar plates without supplemented antimicrobials were screened by multiplex PCR for the detection of the stx 1 , st $x 2$ and aggR genes. One chicken farm sample each $(0.5 \%)$ contained the $s t \times 1$ and the aggR gene. Eleven (2.4\%) human faecal samples contained the stx 1 gene, 2 samples $(0.4 \%)$ contained stx2 gene, and 31 (6.8 \%) contained the aggR gene. From 46 PCR-positive samples, 205 E. coli isolates were tested for the presence of $s t \times 1, s t \times 2$, aggR, $w_{Z X} X_{104}$ and fli $C_{H 4}$ genes. None of the isolates simultaneously contained the four genetic markers associated with E. coli O104:H4 epidemic strain (aggR, stx2, wZX 0104 and fli $_{\mathrm{H}_{4}}$ ). Of 34 EAEC, $64.7 \%$ were resistant to $3^{\text {rd }}$-generation cephalosporins.

Conclusion: These results indicate that in southern Vietnam, the human population is a more likely reservoir of aggR and stx gene carrying E. coli than the chicken population. However, conditions for transmission of isolates and/or genes between human and animal reservoirs resulting in the emergence of highly virulent $E$. coli strains are still favorable, given the nature of'backyard' farms in Vietnam.
\end{abstract}

Keywords: EAEC, STEC, E. coli, Chicken, Humans, Vietnam

\footnotetext{
* Correspondence: trungnv@oucru.org

'Department of Medical Microbiology, Academic Medical Center, University

of Amsterdam, Amsterdam, The Netherlands

2Department of Global Health-Amsterdam Institute for Global Health and

Development, Amsterdam, The Netherlands

Full list of author information is available at the end of the article
} 


\section{Background}

Escherichia coli is one of the most widely distributed bacteria in the environment, and therefore humans and animals are exposed to saprophytic E. coli strains throughout their lives. Some E. coli strains may become capable of causing disease in humans and some animal species by expression of one or multiple virulence factors, such as adhesins and toxins [1]. These virulence factors are encoded by genes which are typically located on mobile genetic elements such as plasmids or phages. Horizontal gene transfer between $E$. coli strains carrying different virulence determinants may result in novel virulence gene combinations leading to highly virulent phenotypes.

An example of the emergence of highly virulent pathogenic E. coli strains is the Enterohaemorrhagic E. coli (EHEC) O104:H4, responsible for the large and devastating outbreak of hemorrhagic colitis in Europe in 2011 [2]. This outbreak strain was found to carry an unusual combination of the pathogenic features of enteroaggregative E. coli (EAEC) and Shiga toxin (Stx)-producing $E$. coli (STEC). Among the 6 pathotypes of E. coli capable of causing enteric diseases, EAEC has emerged as a cause of acute and persistent diarrhea in children, as well as adults, in both developed and developing countries $[3,4]$. EAEC has rarely been isolated from animal sources such as dogs and cats [5], but whether animals are reservoirs of EAEC or are accidental hosts of EAEC due to close contact with humans [6] remains to be determined. In contrast, it has been shown that cattle are the major reservoir species of STEC. However, other livestock species, including sheep, goats, horses, pigs, and water buffalo, are also capable of harboring these organisms [7]. In fact, STEC has been found in contaminated food, water [8] and the farm environment [9-11]. Given these differences between reservoirs, it is striking that Stx-producing EAEC has emerged to cause outbreaks in humans [12]. Therefore, further investigation of the distribution of genes encoding for virulence factors $a g g R$, stx 1 and stx 2 in environmental, animal and human reservoirs is required to better understand the potential for the emergence of $E$. coli carrying this unusual combination of virulence genes.

Although the prevalence of STEC in chicken is considered minimal, a previous study has shown that chickens are readily and persistently infected by STEC [13]. Small-scale and backyard chicken farming are very common in southeast Asia, including the Mekong Delta in southern Vietnam $[14,15]$. In such farms, there is typically a great degree of overlap between the farming and household environments, providing ample opportunity for horizontal gene transfer between human and poultry E. coli. We aimed to assess the potential of such an occurance, by investigating the prevalence of the highly conserved $a g g R$ and the stx1, stx 2 genes in samples collected from chicken farms, farmers and in matched asymptomatic humans not exposed to chickens, as well as in $E$. coli isolates from these samples. In addition, with the hypothesis that E. coli O104:H4 epidemic strains may be present in Vietnam, we also screened for the presence of the combination of $\operatorname{aggR}, s t x 2, w z x_{\mathrm{O} 104}$ and $\mathrm{fliC}_{\mathrm{H} 4}$ genes in $\mathrm{E}$. coli isolates.

\section{Methods}

\section{Sample collection}

This study was carried out as a part of a larger study on antimicrobial drug usage and antimicrobial resistant $E$. coli colonization in backyard chicken farms and humans. We collected faecal swabs from 204 randomly selected chicken farms; 204 healthy farmers in those farms, and 306 age- and sex-matched individuals in Tien Giang province, Vietnam. The matched individuals not involved in poultry farming were randomly selected from the same district as the farmers (rural individual, $N=204$,), as well as from the provincial capital (urban individual, $N=102$ ), using the population census of Tien Giang [16]. Farms and household visits were evenly distributed over a 13month-period from March 2012 to April 2013 in order to avoid seasonal effects. Faecal samples from chickens were collected using boot-swabs or hand-held gauze swabs, as described previously [17]. The chicken sample collection was conducted by a trained sampling team from the Tien Giang Sub-Department of Animal Health. Rectal swab samples were obtained from all human participants by trained staff from Tien Giang Preventive Medicine Center, using Fecalswab (Copan, Italy). All samples were stored and transported at $4 \mathrm{oC}$ to the laboratory at the Oxford University Clinical Research Unit in Ho Chi Minh City and cultured within $24 \mathrm{~h}$ after sample collection.

\section{E. coli isolation and antimicrobial susceptibility testing}

Buffered Peptone Water (225 mL) was added to each chicken faecal sample in a separate container and was manually shaken. A volume of $1 \mathrm{~mL}$ from each container was diluted 1:1000 in saline solution. Human rectal swabs were vortexed to release and suspend the sample in the liquid transport medium and then $100 \mu \mathrm{L}$ was diluted 1:100 in saline solution. A volume of $50 \mu \mathrm{L}$ of each saline diluted sample was plated onto MacConkey agar (Oxoid, UK) with or without antimicrobials supplemented. This procedure yielded on average 100 single colonies on each plate, which allowed for unbiased detection of $E$. coli-like colonies of multiple morphologies in all samples. A random selection of 5 (from MacConkey agar plate without antimicrobial supplemented) and 2 (from each of MacConkey agar plates supplemented with either nalidixic acid [16 mg/L], ceftazidime $[2 \mathrm{mg} / \mathrm{L}]$, or gentamicin $[8 \mathrm{mg} / \mathrm{L}])$ presumptive E. coli-like colonies were sub-cultured, identified 
and tested for their susceptibility using disc diffusion method in accordance with the Clinical and Laboratory Standards Institute guidelines [18]. Eleven antimicrobials (all Oxoid, UK) were tested including tetracycline $(30 \mathrm{mg})$, trimethoprim/sulfamethoxazole $(1.25 / 23.75 \mathrm{mg})$, chloramphenicol (30 mg), gentamicin (10 mg), amikacin (30 mg), ciprofloxacin $(5 \mathrm{mg})$, ampicillin (10 mg), amoxicillin/clavulanic acid (30 mg), ceftazidime (30 mg), ceftriaxone (30 mg) and meropenem (10 mg). Quality controls for susceptibility testing and identification were performed every week according to the CLSI guidelines. Strains with an intermediate susceptible result were considered resistant. An MDR strain was defined as a strain resistant to at least three different antimicrobial classes.

Only E. coli strains with a unique morphology and/or phenotypic antimicrobial susceptibility pattern isolated from each sample were saved for further analyses. In addition, a sweep from the full remaining growth on the MacConkey plate without antimicrobial supplement was collected, suspended in glycerol and stored in screw-cap tubes at $-20{ }^{\circ} \mathrm{C}$ for further analyses.

\section{Screening for the presence of $E$. coli virulence factors}

As using sweep samples for PCR screening has been shown to be efficient and sensitive [19], multiplex PCR was first performed on the glycerol stored sweeps to screen for the presence of $a g g R, s t x 1$ and $s t x 2$ genes. In brief, one loopful $(1 \mu \mathrm{l})$ of the $-20{ }^{\circ} \mathrm{C}$ stored sweep was collected and cultured on the MacConkey agar, then incubated for $16 \mathrm{~h}$ at $37{ }^{\circ} \mathrm{C}$. A sweep of the bacterial growth was then collected and suspended in $1 \mathrm{ml}$ of water. Suspensions were heated at $95{ }^{\circ} \mathrm{C}$ for $3 \mathrm{~min}$ and immediately placed on ice then centrifuged at $9000 \mathrm{rpm} / 3 \mathrm{~min}$ to collect DNA in the supernatant. Primers used for the multiplex PCR are listed in Table 1. Primers were designed by using Nucleotide Blast, Align $\mathrm{X}$ and OligoAnalyzer 3.1 with the published sequences on NCBI database (Genbank accession numbers are M19473, X07865, Z18751 for stx1, stx2 and aggR, respectively). The detection limit of the assay was determined by cloning the target sequences into a pCR2.1 plasmid (TA Cloning kit, Invitrogen, USA). The PCR had a detection limit of 10 copies per $\mathrm{ml}$ for each of the target gene as determined by serial dilution from $10 \mathrm{ng} / \mu \mathrm{l}$ to $10^{-6} \mathrm{ng} / \mu \mathrm{l}$. Each reaction mixture contained $2 \mathrm{mM}$ $\mathrm{MgCl}_{2}, 0.1 \mathrm{mM}$ deoxyribonucleotides, $0.2 \mathrm{uM}$ each of the oligonucleotides and $0.5 \mathrm{U}$ of Taq polymerase (Bioline, UK), to which $50-100 \mathrm{ng}$ of the DNA suspension was added. The mixtures were processed in a GeneAmp PCR system 9700 (Applied Biosystems, USA). The PCR program was $96^{\circ} \mathrm{C}$ for $4 \mathrm{~min}, 30$ cycles of $94{ }^{\circ} \mathrm{C}$ for $20 \mathrm{~s}$, annealing at $55^{\circ} \mathrm{C}$ for $20 \mathrm{~s}$, and $72{ }^{\circ} \mathrm{C}$ for $10 \mathrm{~s}$. The final extension step was $72{ }^{\circ} \mathrm{C}$ for $7 \mathrm{~min}$. DNA extracted from E. coli E3787 (stx1), E. coli E32511 (stx2), E. coli E69187 $(a g g R)$ was used as a positive control in PCR reactions. A negative control, containing water instead of template DNA was included in each run of PCR reactions.

From any sweep sample which produced positive PCR result for any of the target genes in multiplex PCR, DNA extracted from all stored E. coli isolates obtained after culture of the corresponding faecal sample on MacConkey agar plates with and without antimicrobials supplemented, was subsequently tested for the presence of 3 genes (aggR, stx 1 and stx2) using multiplex PCR. The presence of 2 genes $\left(w z x_{\mathrm{O} 104}\right.$ and $\left.f l i \mathrm{C}_{\mathrm{H} 4}\right)$ was also investigated using monoplex PCR [20].

\section{Results}

Sweep samples were available for 188 of 204 chicken farms, 186 of 204 farmers, 182 of 204 rural individuals and 90 of 102 urban individuals. The remaining sweep samples were either missing (16 chicken samples and 45 human samples) or were not available because the primary faecal culture on MacConkey agar did not show any growth (7 human samples). The aggR gene was detected in $31(6.8 \%)$ human samples but in only $1(0.5 \%)$ of the chicken farm samples $(p<0.001)$. Gene stx 1 was detected in 11 human samples $(2.4 \%)$ and in one chicken

Table 1 List of primers used in this study

\begin{tabular}{|c|c|c|c|c|}
\hline Target genes & Primer name & Primer sequences $\left(5^{\prime}-3^{\prime}\right)$ & Fragment size & Reference \\
\hline \multirow[t]{2}{*}{$\operatorname{aggR}$} & aggR_F & AAGCAGCGATACATTAAGACG & 424 & This study \\
\hline & aggR_R & TGCTITGCTCATTCTTGATTGC & & \\
\hline \multirow[t]{2}{*}{ stx1 } & stx1_F & TGATGATTGATAGTGGCACAGG & 299 & This study \\
\hline & $s t \times 1 \_R$ & AGAAGTAGTCAACGAATGGCG & & \\
\hline \multirow[t]{2}{*}{$s t \times 2$} & stx2_F & ACATCGGTGTCTGTTATTAACC & 666 & This study \\
\hline & $s t \times 2 \_R$ & TTGACTCTCTTCATTCACGGC & & \\
\hline \multirow[t]{2}{*}{$w Z X_{O 104}$} & $W Z X_{0104 \_} F$ & GGTTITATTGTCGCGCAAAG & 337 & [20] \\
\hline & WZX $104 \_R$ & TATGCTCTIITTCCCCATCG & & \\
\hline \multirow[t]{2}{*}{$\mathrm{fliC}_{\mathrm{H} 4}$} & $\mathrm{fliC}_{\mathrm{H} 4 \_} \mathrm{F}$ & ACGGCTGCTGATGGTACAG & 244 & [20] \\
\hline & $\mathrm{fliC}_{\mathrm{H} 4 \_\mathrm{R}}$ & CGGCATCCAGTGCTITTAAC & & \\
\hline
\end{tabular}


farm sample $(0.5 \%)$ whereas gene stx 2 was detected in one farmer $(0.5 \%)$ and in one rural individual (0.5 \%). In 46 samples, at least one of three tested genes (aggR, stx 1 and $s t x 2)$ was detected in the multiplex PCR. None of the samples was positive with multiple genes (Table 2).

DNA samples of $205 \mathrm{E}$. coli isolated from those 46 MacConkey sweep samples were tested for the presence of $\operatorname{aggR}, s t x 1, s t x 2$ and then further screened for the presence of $w z x_{\mathrm{O} 104}$ and $f l i C_{\mathrm{H} 4}$ gene. The aggR gene was detected in 29.1, 27.6 and 7.4\% of E. coli strains isolated from aggR positive samples in farmers, rural individuals and urban individuals, respectively (Table 2). EAEC could be isolated from faecal samples of $3.8 \%$ (7/186) of farmers, $3.8 \%$ (7/182) rural individuals and $1.1 \%(1 / 90)$ urban individuals. The EAEC isolates exhibited resistance against ampicillin (100\%), co-trimoxazole (85.3\%), tetracycline (70.6\%), gentamicin (70.6\%), ceftriaxone (64.7\%), ceftazidime (50.0\%), chloramphenicol (38.2 \%), ciprofloxacin (26.5\%) and amikacin (2.9\%). $88.2 \%$ and $50.0 \%$ of EAEC isolates were multidrug resistant and extended-spectrum beta-lactamase positive, respectively (Table 3 ).

From the 12 stx 1 positive sweep samples, only one $E$. coli $(3.4 \%)$ from a farmer, among $53 \mathrm{E}$. coli isolates investigated, was $s t x 1$ positive (Table 2). This $E$. coli isolate was also multi-drug resistant against chloramphenicol, sulfamethoxazole-trimethoprim, ampicillin, tetracycline and ciprofloxacin). Detailed information on antimicrobial resistance patterns of EAEC and STEC isolated from humans in southern Vietnam is shown in Additional file 1: Table S1. Eight $E$. coli isolates from the two $s t x 2 \mathrm{PCR}$ positive samples were tested and none showed a positive result for $s t x 2$ gene. As a result, STEC was isolated in $0.5 \%(1 / 186)$ of farmers or $0.2 \%(1 / 458)$ of studied humans.

Among 196 E. coli isolates from 44 human individuals that were positive for any of the genes screened for using the multiplex PCR, $\mathrm{fliC}_{\mathrm{H} 4}$ gene was detected in 13.6, 6.7 and $3.0 \%$ of $E$. coli isolates from farmers, rural individuals and urban individuals, respectively (Table 2). One out of 9 isolates from two farm samples $(11.1 \%)$ was positive with $f l i C_{\mathrm{H} 4}$ gene. Gene $w z x_{0104}$ was observed in one isolate from a farmer $(1.1 \%)$. The only gene combination that we

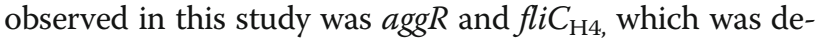
tected in two isolates from one rural individual. None of the isolates analyzed in our study simultaneously contained the four genetic markers associated with the O104:H4 epidemic strain $\left(a g g R, s t x 2, w z x_{\mathrm{O} 104}\right.$ and $\left.f l i C_{\mathrm{H} 4}\right)$.

\section{Discussion}

The differences in detection of the $\operatorname{aggR}$ gene in chicken faecal samples $(1 ; 0.5 \%)$ and samples from asymptomatic humans $(31 ; 6.8 \%)$ suggest that humans are likely the main reservoir of EAEC in the setting studied. The isolation rate of EAEC from humans in our study (3.3\%, 15/458) was similar to previous studies including asymptomatic adults and children performed in northern Vietnam [21, 22]. To our knowledge, this is the first study describing the presence of the aggR gene in chicken faecal samples in Vietnam. We, however, speculate that this gene may have a human origin since a relatively high proportion of households in the rural areas of the Mekong Delta do not have latrines that meet established hygiene standards with respect to their construction, operation and maintenance [23].

Gene $s t x 1$ was also detected more frequently in samples from humans compared with those from chickens $(2.4 \%$ versus $0.5 \%$ ) and the more toxic gene-stx 2 -was only detected in two samples from humans $(0.4 \%)$. The nondetection of STEC in chicken farms is in agreement with previous studies in chickens in the United States and United Kingdom in which the prevalence of STEC colonization ranged between 0 and $1.5 \%$ [24]. Although we did not find the presence of STEC in chicken samples in this study, STEC is still an important cause of diarrhea in farm animals in Vietnam as has been shown in previous studies $[25,26]$. The isolation rate of STEC $(0.2 \%, 1 / 458)$ and EAEC ( $3.3 \%$, $15 / 458$ ) in asymptomatic humans in Vietnam is also similar to the reported prevalence in both developed and developing countries [27-29]. Our results indicate a generally high level of antimicrobial resistance among EAEC isolates from Vietnam compared with results from other countries [30] and overuse of antimicrobials in the community could be one of the possible explanations [31].

Table 2 Prevalence of aggR, stx 1 and stx2 genes in sweep samples and prevalence of aggR, stx1, stx2, wZX $x_{0104}$ and fli $C_{\mathrm{H} 4} g e n e s$ in $E$. coli isolated from chicken faecal samples and human rectal swabs

\begin{tabular}{|c|c|c|c|c|c|c|c|c|}
\hline \multirow[t]{2}{*}{ Subject } & \multicolumn{3}{|c|}{ No. of positive samples (\%) } & \multicolumn{5}{|c|}{ No. of positive E. coli / No. of E. coli investigated (\%) } \\
\hline & $\operatorname{agg} R$ & stxl & $s t \times 2$ & $\operatorname{agg} R$ & $s t \times 1$ & $s t \times 2$ & $W Z X_{0104}$ & $\mathrm{fli}_{\mathrm{H} 4}$ \\
\hline Chicken farm $(N=188)$ & $1(0.5)$ & $1(0.5)$ & 0 & $0 / 4$ & $0 / 5$ & ND & $0 / 9$ & $1 / 9(11.1)$ \\
\hline Farmer $(N=186)$ & $12(6.5)$ & $7(3.8)$ & $1(0.5)$ & $16^{\mathrm{a}} / 55(29.1)$ & $1 / 29(3.4)$ & $0 / 4$ & $1 / 88(1.1)$ & 12/88 (13.6) \\
\hline Rural individual $(N=182)$ & $13(7.1)$ & $3(1.6)$ & $1(0.5)$ & $16^{\mathrm{b}} / 58(27.6)$ & $0 / 13$ & $0 / 4$ & $0 / 75$ & $5 / 75(6.7)$ \\
\hline Urban individual $(N=90)$ & $6(6.7)$ & $1(1.1)$ & 0 & $2^{c} / 27(7.4)$ & $0 / 6$ & ND & $0 / 33$ & 1/33 (3.0) \\
\hline
\end{tabular}

a aggR-positive E. coli were isolated from samples of 7 farmers

${ }^{b}$ aggR-positive $E$. coli were isolated from samples of 7 rural individuals

caggR-positive E. coli were isolated from sample of 1 urban individual 
Table 3 Antimicrobial susceptibility of EAEC isolates from asymptomatic humans in southern Vietnam

\begin{tabular}{|c|c|c|c|c|}
\hline \multirow[t]{3}{*}{ Antimicrobial } & \multicolumn{4}{|c|}{ No. of antimicrobial resistant EAEC (\%) } \\
\hline & Chicken farmer & Rural individual & Urban individual & Total \\
\hline & $(N=16)$ & $(N=16)$ & $(N=2)$ & $(N=34)$ \\
\hline Tetracycline & $8(50.0)$ & $14(87.5)$ & $2(100)$ & $24(70.6)$ \\
\hline Trimethoprim/sulfamethoxazole & $13(81.2)$ & $14(87.5)$ & $2(100)$ & $29(85.3)$ \\
\hline Chloramphenicol & $11(68.8)$ & $2(12.5)$ & 0 & $13(38.2)$ \\
\hline Gentamicin & $13(81.2)$ & $11(68.8)$ & 0 & $24(70.6)$ \\
\hline Amikacin & 0 & $1(6.2)$ & 0 & $1(2.9)$ \\
\hline Ciprofloxacin & 0 & $9(56.2)$ & 0 & $9(26.5)$ \\
\hline Ampicillin & $16(100)$ & $16(100)$ & $2(100)$ & $34(100)$ \\
\hline Amoxicillin/clavulanic acid & $8(50.0)$ & $7(43.8)$ & $2(100)$ & $17(50.0)$ \\
\hline Ceftazidime & $6(37.5)$ & $11(68.8)$ & 0 & $17(50.0)$ \\
\hline Ceftriaxone & $8(50.0)$ & $14(87.5)$ & 0 & $22(64.7)$ \\
\hline Third-generation cephalosporins & $8(50.0)$ & $14(87.5)$ & 0 & $22(64.7)$ \\
\hline ESBL-producing & $6(37.5)$ & $11(68.8)$ & 0 & $17(50.0)$ \\
\hline Meropenem & 0 & 0 & 0 & 0 \\
\hline MDR & $13(81.2)$ & $15(93.8)$ & $2(100)$ & $30(88.2)$ \\
\hline
\end{tabular}

MDR multi-drug resistant

A total of $150(73.2 \%)$ out of 205 E. coli isolates, which were isolated from positive sweep samples, were negative in the colony PCR for detection of the five genes of interest. The lower isolation rate of EAEC and STEC in comparison to the stx and aggR gene detection rate on sweep samples may be due to the presence of the $s t x$ or aggR genes in E. coli strains other than those selected or in bacterial species other than $E$. coli but capable of growing on MacConkey agar [32]. In addition, the bacterial sweep was used as it was previously shown to better detect $E$. coli in stool samples than testing up to $5 \mathrm{E}$. coli isolates because of its enrichment [19]. Hence differences in sensitivity can also explain lower detection rates when testing single colonies and the absence of stx-positive $E$. coli amongst the limited number of isolates tested, does not completely rule out the presence of STEC in the sample.

The full combination of the four typical markers of the 2011 German E. coli O104:H4 outbreak strain stx2, $w z x_{\mathrm{O} 104}, f l i C_{\mathrm{H} 4}$ and $a g g R$ was not detected in any E. coli isolated from human faecal and chicken farm samples in the current study, despite the great degree of overlap between the farming and living environment in the study setting in Vietnam. We found one combination of EAEC and STEC O104:H4 associated $\operatorname{aggR}$ and $f l i \mathrm{C}_{\mathrm{H} 4}$ genes in two $E$. coli isolates from one rural individual not exposed to poultry farming.

\section{Conclusions}

Our results indicate that in southern Vietnam, the human population is a more likely reservoir of $a g g R$ and stx gene carrying E. coli than the chicken population. However, it is important to note that all four typical markers of the outbreak strain $\left(s t x 2, w z x_{\mathrm{O} 104}, f l i C_{\mathrm{H} 4}\right.$ and $\operatorname{aggR}$ ) were detected, albeit at low numbers, in samples (stx 2 and $a g g R)$ and $E$. coli isolates $\left(w z x_{\mathrm{O} 104}, f l i C_{\mathrm{H} 4}\right)$ in different host populations. Given the nature of 'backyard' farms in Vietnam, conditions for transmission of isolates and/or genes between human and animal reservoirs are still favorable. Therefore, strict personal hygiene practices as well as applying biosecurity in animal farming are essential to avoid the emergence of highly virulent strains.

\section{Additional file}

Additional file 1: Table S1. Patterns of antimicrobial resistance in EAEC and STEC isolated from humans in southern Vietnam. (XLSX $10 \mathrm{~kb}$ )

\section{Abbreviations}

EAEC: Enteroaggregative Escherichia coli; EHEC: Enterohaemorrhagic Escherichia coli; PMC: Preventive Medicine Centre of Tien Giang; SDAH: Sub-Department of Animal Health of Tien Giang; STEC: Shiga toxin (Stx)-producing Escherichia coli

\section{Acknowledgements}

We would like to thank the staff at the Preventive Medicine Center and Sub-Department of Animal Health in Tien Giang for their support in the sample collection.

Funding

This work was funded by ZonMW/WOTRO, the Netherlands (project number 205100012), the EU-FP7 program ANTIGONE (project number 278976) and The Wellcome Trust, UK (089276/Z/09/Z). 


\section{Availability of data and materials}

The data supporting the antimicrobial susceptibility results is contained in the Additional file 1.

\section{Authors' contributions}

NVT, HNN, CS and NTH participated in the design of the study; HNN, NTN, HTT and PVM carried out microbiological analysis including PCR and susceptibility testing; $\mathrm{HHM}, \mathrm{TQH}$ and NTNM contributed to the acquisition of microbiological data; CS, NTH and JC contributed reagents/materials/analysis tools. JJC, JW, CS, NTH, HNN, NVT participated in the data analysis and interpretation of data. NVT, HNN drafted the manuscript. All authors read and approved the final manuscript.

\section{Competing interests}

The authors declare that they have no competing interests.

\section{Consent for publication}

Not applicable.

\section{Ethics approval and consent to participate}

Written informed consent was obtained from all participants prior to inclusion in the study. Written consent was also obtained from farmers before collecting chicken samples. Sub-Department of Animal Health in Tien Giang approved the animal sampling procedures. The study procedure was also approved by the Peoples' Committee of Tien Giang Province. Ethical approval for this study with respect to humans was granted by the Department of Health in Tien Giang and the Oxford University Tropical Research Ethics Committee (OxTREC, No. 48/11).

\section{Author details}

'Department of Medical Microbiology, Academic Medical Center, University of Amsterdam, Amsterdam, The Netherlands. ${ }^{2}$ Department of Global Health-Amsterdam Institute for Global Health and Development, Amsterdam, The Netherlands. ${ }^{3}$ Oxford University Clinical Research Unit, Centre for Tropical Medicine, Ho Chi Minh City, Vietnam. ${ }^{4}$ Centre for Tropical Medicine, Nuffield Department of Medicine, University of Oxford, Oxford, UK. ${ }^{5}$ Sub-Department of Animal Health, My Tho, Tien Giang, Vietnam. ${ }^{6}$ Department of Infectious Diseases and Immunology, Faculty of Veterinary Medicine, Utrecht University, Utrecht, The Netherlands. ${ }^{7}$ Central Veterinary Institute of Wageningen UR, Lelystad, The Netherlands. ${ }^{8}$ Preventive Medicine Center, My Tho, Tien Giang, Vietnam.

\section{Received: 20 February 2016 Accepted: 31 August 2016} Published online: 09 September 2016

\section{References}

1. Croxen MA, Law RJ, Scholz R, Keeney KM, Wlodarska M, Finlay BB. Recent advances in understanding enteric pathogenic Escherichia coli. Clin Microbiol Rev. 2013;26(4):822-80.

2. Rasko DA, Webster DR, Sahl JW, Bashir A, Boisen N, Scheutz F, et al. Origins of the E. coli strain causing an outbreak of hemolytic-uremic syndrome in Germany. N Engl J Med. 2011;365(8):709-17.

3. Huang DB, Nataro JP, DuPont HL, Kamat PP, Mhatre AD, Okhuysen PC, et al. Enteroaggregative Escherichia coli is a cause of acute diarrheal illness: a meta-analysis. Clin Infect Dis. 2006;43(5):556-63.

4. Okhuysen PC, Dupont HL. Enteroaggregative Escherichia coli (EAEC): a cause of acute and persistent diarrhea of worldwide importance. J Infect Dis. 2010;202(4):503-5.

5. Puno-Sarmiento J, Medeiros L, Chiconi C, Martins F, Pelayo J, Rocha S, et al. Detection of diarrheagenic Escherichia coli strains isolated from dogs and cats in Brazil. Vet Microbiol. 2013;166(3-4):676-80.

6. Johnson JR, Owens K, Gajewski A, Clabots C. Escherichia coli colonization patterns among human household members and pets, with attention to acute urinary tract infection. J Infect Dis. 2008;197(2):218-24.

7. Ferens WA, Hovde CJ. Escherichia coli O157:H7: animal reservoir and sources of human infection. Foodborne Pathog Dis. 2011;8(4):465-87.

8. Scallan E, Hoekstra RM, Angulo FJ, Tauxe RV, Widdowson MA, Roy SL, et al. Foodborne illness acquired in the United States-major pathogens. Emerg Infect Dis. 2011;17(1):7-15.

9. Islam M, Doyle MP, Phatak SC, Millner P, Jiang X. Persistence of enterohemorrhagic Escherichia coli O157:H7 in soil and on leaf lettuce and parsley grown in fields treated with contaminated manure composts or irrigation water. J Food Prot. 2004;67(7):1365-70.

10. Solomon EB, Yaron S, Matthews KR. Transmission of Escherichia coli O157:H7 from contaminated manure and irrigation water to lettuce plant tissue and its subsequent internalization. Appl Environ Microbiol. 2002;68(1):397-400.

11. Doane CA, Pangloli P, Richards HA, Mount JR, Golden DA, Draughon FA. Occurrence of Escherichia coli 0157:H7 in diverse farm environments. J Food Prot. 2007;70(1):6-10.

12. Grad YH, Lipsitch M, Feldgarden M, Arachchi HM, Cerqueira GC, Fitzgerald $\mathrm{M}$, et al. Genomic epidemiology of the Escherichia coli O104:H4 outbreaks in Europe, 2011. Proc Natl Acad Sci U S A. 2012;109(8):3065-70.

13. La Ragione RM, Best A, Sprigings K, Liebana E, Woodward GR, Sayers AR, et al. Variable and strain dependent colonisation of chickens by Escherichia coli O157. Vet Microbiol. 2005;107(1-2):103-13.

14. Burgos S, Hong Hanh PT, Roland-Holst D, Burgos SA. Characterization of poultry production systems in Vietnam. Int J of Poult Sci. 2007;6(10):709-12.

15. Desvaux S, Ton VD. A general review and description of the poultry production in Vietnam. In: Research consortium on risks associated with livestock intensification. Hanoi: CIRAD; 2008.

16. Statistical Office of Tien Giang Province. Statistical YearBook Tien Giang Province 2011. Ho Chi Minh city: Statistical Printing Factory; 2012.

17. Nguyen VT, Carrique-Mas JJ, Ngo TH, Ho HM, Ha TT, Campbell Jl, et al. Prevalence and risk factors for carriage of antimicrobial-resistant Escherichia coli on household and small-scale chicken farms in the Mekong Delta of Vietnam. J Antimicrob Chemother. 2015;70(7):2144-52.

18. CLSI. Performance standards for antimicrobial susceptibility testing; twentyfirst international supplement M100-S21. Wayne: Clinical and Laboratory Standards Institute; 2011.

19. Schultsz C, Pool GJ, van Ketel R, de Wever B, Speelman P, Dankert J. Detection of enterotoxigenic Escherichia coli in stool samples by using nonradioactively labeled oligonucleotide DNA probes and PCR. J Clin Microbiol. 1994;32(10):2393-7.

20. Paddock ZD, Bai J, Shi X, Renter DG, Nagaraja TG. Detection of Escherichia coli $\mathrm{O} 104$ in the feces of feedlot cattle by a multiplex PCR assay designed to target major genetic traits of the virulent hybrid strain responsible for the 2011 German outbreak. Appl Environ Microbiol. 2013;79(11):3522-5.

21. Do TT, Bui TT, Molbak K, Phung DC, Dalsgaard A. Epidemiology and aetiology of diarrhoeal diseases in adults engaged in wastewater-fed agriculture and aquaculture in Hanoi, Vietnam. Trop Med Int Health. 2007;12 Suppl 2:23-33.

22. Hien BT, Scheutz F, Cam PD, Serichantalergs O, Huong TT, Thu TM, et al. Diarrheagenic Escherichia coli and Shigella strains isolated from children in a hospital case-control study in Hanoi, Vietnam. J Clin Microbiol. 2008;46(3):996-1004

23. Van Minh H, Nguyen-Viet $H$, Thanh NH, Yang JC. Assessing willingness to pay for improved sanitation in rural Vietnam. Environ Health Prev Med. 2013;18(4):275-84

24. Persad AK, LeJeune JT. Animal reservoirs of Shiga toxin-producing Escherichia coli. Microbiol Spectr. 2014;2(4):EHEC-0027-2014.

25. Nguyen TD, Vo TT, Vu-Khac H. Virulence factors in Escherichia coli isolated from calves with diarrhea in Vietnam. J Vet Sci. 2011;12(2):159-64.

26. Vu-Khac H, Cornick NA. Prevalence and genetic profiles of Shiga toxinproducing Escherichia coli strains isolated from buffaloes, cattle, and goats in central Vietnam. Vet Microbiol. 2008;126(4):356-63.

27. Nataro JP, Mai V, Johnson J, Blackwelder WC, Heimer R, Tirrell S, et al. Diarrheagenic Escherichia coli infection in Baltimore, Maryland, and New Haven, Connecticut. Clin Infect Dis. 2006;43(4):402-7.

28. Keskimaki M, Eklund M, Pesonen H, Heiskanen T, Siitonen A, Study G. EPEC, EAEC and STEC in stool specimens: prevalence and molecular epidemiology of isolates. Diagn Microbiol Infect Dis. 2001;40(4):151-6.

29. Opintan JA, Bishar RA, Newman MJ, Okeke IN. Carriage of diarrhoeagenic Escherichia coli by older children and adults in Accra, Ghana. Trans R Soc Trop Med Hyg. 2010;104(7):504-6.

30. Kong H, Hong X, Li X. Current perspectivesin pathogenesis and antimicrobial resistance of enteroaggregative Escherichia coli. Microb Pathog. 2015;85:44-9.

31. Nga do TT, Chuc NT, Hoa NP, Hoa NQ, Nguyen NT, Loan HT, et al. Antibiotic sales in rural and urban pharmacies in northern Vietnam: an observational study. BMC Pharmacol Toxicol. 2014;15:6.

32. Mauro SA, Koudelka GB. Shiga toxin: expression, distribution, and its role in the environment. Toxins (Basel). 2011;3(6):608-25. 